\title{
A search asymmetry for interocular conflict
}

\author{
Chris L. E. Paffen • Ignace T. C. Hooge • \\ Jeroen S. Benjamins • Hinze Hogendoorn
}

Published online: 19 February 2011

(C) The Author(s) 2011. This article is published with open access at Springerlink.com

\begin{abstract}
When two different images are presented to the two eyes, the percept will alternate between the images (a phenomenon called binocular rivalry). In the present study, we investigate the degree to which such interocular conflict is conspicuous. By using a visual search task, we show that search for interocular conflict is near efficient (15 ms/item) and can lead to a search asymmetry, depending on the contrast in the display. We reconcile our findings with those of Wolfe and Franzel (1988), who reported inefficient search for interocular conflict (26 ms/item) and found no evidence for a search asymmetry. In addition, we provide evidence for the suggestion that differences in search for interocular conflict are contingent on the degree of abnormal fusion of the dissimilar images.
\end{abstract}

Keywords Binocular rivalry . Visual attention .

Visual search $\cdot$ Interocular conflict

\section{Introduction}

Since the eyes are separated by about $6 \mathrm{~cm}$, each of the retinas receives a different projection of the visual world. Due to these different projections, images can only be fused within Panum's area (Panum, 1858). As a result, the visual system is provided with conflicting information about a single visual scene. Interestingly, we are usually unaware of the conflict,

C. L. E. Paffen $(\varangle) \cdot$ I. T. C. Hooge $\cdot$ J. S. Benjamins $\cdot$

H. Hogendoorn

Helmholtz Institute and Department of Experimental Psychology,

Utrecht University,

Heidelberglaan 2,

3584 CS Utrecht, The Netherlands

e-mail: c.l.e.paffen@uu.nl although it can be observed easily. ${ }^{1}$ Another way of inducing conflict in images projected to the retinas is by presenting a different image to each of the two eyes (e.g., images of a house and a face). When an observer views such a stimulus, perception will alternate between the two images. This phenomenon is referred to as binocular rivalry (for reviews, see Alais \& Blake, 2005; Blake \& Logothetis, 2002). When such a conflict is induced, an observer is immediately aware that the eyes are presented with conflicting information.

It is apparent that the presence of conflicting information on the two retinas will be noticed in some cases (during binocular rivalry), but not in others (during "normal" binocular vision). Although there are notable differences between the conflict during normal vision and when experiencing binocular rivalry (we will return to this in the General discussion), here we ask the question: How conspicuous is the conflict between different images presented to the two eyes?

To investigate the degree of conspicuity of interocular conflict, we use a well-known paradigm: visual search. During visual search, an observer searches for a target among a variable number of distractors. The degree of conspicuityor salience - of a target can then be assessed by determining how search time is affected by set size (the total number of items - distractors and target[s]). Original models on visual search describe two ways in which search time can vary with set size: one in which search times are independent of set size, and one in which search times increase with the set size. When there is no effect of set size, search has been labeled parallel; when there is an effect of set size, search has been labeled serial (e.g., Neisser, 1967; Treisman \& Gelade, 1980; Wolfe, 1998). There is much discussion about what underlying processes might be responsible for different search perfor-

\footnotetext{
${ }^{1}$ Put one thumb behind the other, just in front of your face. Now fixate on the closest one. If you have accurate stereo vision, you will perceive three thumbs.
} 
mance, since a simple serial-parallel dichotomy is not supported by many experiments. Therefore, it has been proposed to label performance on a scale from efficient, via quite efficient, to inefficient and very inefficient (Wolfe, 1998). Whatever terminology is used to describe search performance, features that can lead to search that is labeled parallel or efficient are said to "pop out," or attract attention, independent of the number of distractors. Such features are conspicuous and are said to be able to guide attention (Wolfe, 1994; Wolfe, Cave, \& Franzel, 1989). Based on visual search studies of the recent decades, several stimulus attributes have been found to be able to guide attention (such as color, motion, orientation, and size; see Wolfe \& Horowitz, 2004, and Wolfe, 1998, for lists). On the other hand, if search time increases with set size and is labeled serial or inefficient, a target does not attract attention, but is found either by making eye movements or scanning a display item-by-item. Finally, a search asymmetry has been observed for some visual attributes. A search asymmetry occurs when search times are affected by reversing the identities of target and distractors. For example, search is faster for a $\mathrm{Q}$ among Os than vice versa (Treisman \& Souther, 1985). Search asymmetries have been observed for stimulus attributes such as orientation, as well as the presence, rather than the absence, of stimulus features (such as the small mark distinguishing a $\mathrm{Q}$ from an O; see Treisman \& Gormican, 1988).

From the discussion of the conspicuity of interocular conflict, two hypotheses can be tested. According to the first hypothesis, interocular conflict is conspicuous. As a result, search will be independent of set size. According to the second hypothesis, such conflict is not conspicuous, and top-down directing of attention is needed in order to find the target during search. In this case, search time will increase with increasing set size. The two hypotheses were tested by using a display in which oriented Gabors were presented in a circular arrangement (see Fig. 1a).

In the first part of the article, we show that search for interocular conflict is near efficient $(15 \mathrm{~ms} /$ item $)$ and that interocular conflict leads to a search asymmetry. In the second part of the article, we reconcile these results with the findings of Wolfe and Franzel (1988), who performed a study very similar to ours but argued that search for interocular conflict is not efficient, nor does it lead to a search asymmetry.

\section{General method}

\section{Observers}

Ten observers participated in Experiments 1, 5, and 7, and 5 observers performed in Experiments 2, 3, 4, 6, and 8. All observers had normal or corrected-to-normal vision. Author C.L.E.P. participated in all experiments; authors H.H. and J.
S.B. participated in Experiments 1, 2, 3, 4 and 5, and the rest of the observers were naïve as to the purpose of the study. An overview of which observer performed in which experiment is given in the Appendix.

\section{Stimulus and apparatus}

Stimuli were computed using an Apple dual 2-GHz PowerPC G5 using MATLAB with Psychophysics Toolbox (Brainard, 1997) extensions and presented on either a linearized LaCie Electron Blue IV 22-in. monitor (Exps. 1-4 and 8) or a linearized 22-in. Iiyama Vision Master Pro 514 (Exps. 5-7). Dichoptic presentation was achieved by using a mirror stereoscope. The stimulus consisted of a variable number of elements presented on an imaginary circle around a fixation point. The elements consisted of oriented (either horizontal or vertical) Gabors. All Gabors had a spatial frequency of $4 \mathrm{cpd}$ and a Gaussian envelope of $0.3^{\circ}$. The peak contrast of the Gabors was $99.5 \%$, and the luminance of the gray background of the monitor was $26.3 \mathrm{~cd} \mathrm{~m}^{-2}$. Elements were centered at a distance of $3^{\circ}$ from the fixation cross. Four high-contrast crosses surrounding the search display, as well as a central fixation cross, were added to both images to aid binocular fusion.

\section{Procedure}

In Experiments 1, 2, 3, 5, 6, 7, and 8, search displays were presented in one of two basic configurations, in which the roles of target and distractor were reversed. The search displays consisted of sets of 3,7 , or 11 elements in Experiments 1, 2, 3 and 8, and sets of 2, 4, and 8 elements in Experiments 5, 6, 7. A total of $50 \%$ of the trials contained a target. In Experiments 1, 2, 3, 5, 6 and 7, observers performed a speeded two-alternative forced choice task, indicating as accurately and rapidly as possible whether or not the display contained a target. In these experiments, the search display was present on the screen until the observer made a response. In Experiment 8, the displays were erased from the screen after $150 \mathrm{~ms}$. In that experiment, observers were simply instructed to indicate whether or not the display contained a target (nonspeeded). Feedback was provided in all of the search experiments. The two basic conditions (in which the roles of targets and distractors were reversed) were tested in separate blocks. Target present and absent trials were intermixed. In all experiments, observers were instructed to refrain from making eye movements and to maintain fixation on the fixation cross.

\section{Data analysis}

In the text, we report mean search times of correct targetpresent trials (although we present data for target-absent 
a

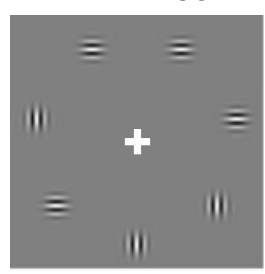

left eye's image

b

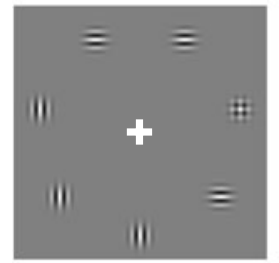

left eye's image

C

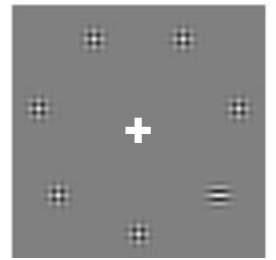

left eye's image conflict target

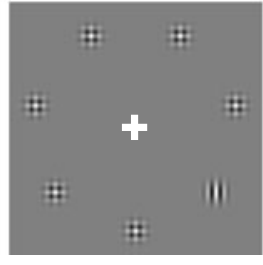

right eye's image

Fig. 1 Stimuli used in Experiments 1, 2, and 3. (a) The stimuli used in Experiment 1. In the conflict target condition (left panel), one of the elements contained Gabor orientations that were orthogonal for the two eyes. In the conflict distractors conditions (right panel), all of the elements except one had orthogonal orientations for the two eyes. (b) The stimuli used in Experiment 2. In the double-target condition (left panel), one of the elements contained two orthogonal orientations. In the double-distractors condition (right panel), all elements except one

trials in the figures). Notably, the accuracy in experiments using reaction times as a measure was $93.6 \%$ or higher. Each data graph shows means of the observers' mean search times. In all experiments, we estimated the slope using linear regression. To evaluate whether a set size effect was present, we tested whether the slopes were significantly different from zero using correlation analysis. Finally, we used a $t$ test to test whether slopes were significantly different.

\section{Experiment 1}

In Experiment 1, we investigated whether interocular conflict is conspicuous by evaluating whether it leads to efficient search. The search displays consisted of two configurations (see Fig. 1a). In the first configuration, the orientation of the target for the left eye was orthogonal to that for the right eye, and the orientations for the distractors

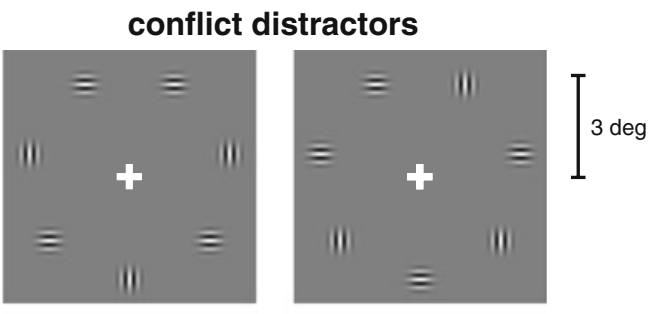

left eye's image

right eye's image

double distractors

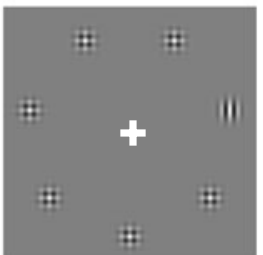

left eye's image

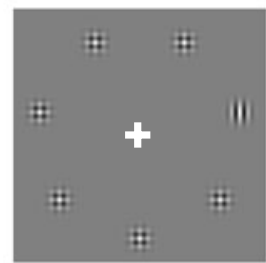

right eye's image conflict distractors

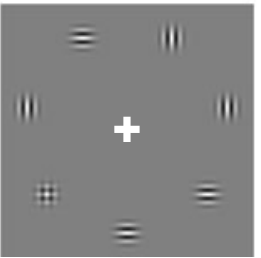

left eye's image

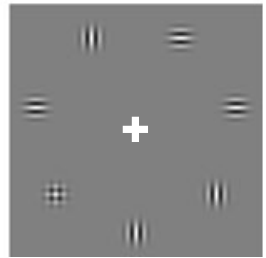

right eye's image contained two orthogonal orientations. (c) The stimuli for Experiment 3. In the conflict target condition (left panel), one of the elements contained Gabor orientations that were orthogonal for the two eyes, while the distractors contained fused orthogonal orientations. In the conflict distractors conditions (right panel), all of the elements except one (which contained fused orthogonal orientations) had orthogonal orientations for the two eyes

were the same for each eye (conflict target condition). In the second configuration, the orientation for the target was the same for the left and the right eyes, and the orientations of the distractors for the left eye were orthogonal to the orientations for the right eye (conflict distractors condition).

\section{Results}

The results of Experiment 1 are displayed in Fig. 2. From the figure, it is clear that search (reflected by reaction times) in the conflict target condition (solid lines) is quite different from that in the conflict distractors condition (dashed lines). The slope of the conflict target condition was significantly lower than that of the conflict distractors condition $[t(18)=$ $2.7, p=.007]$. The slope of the conflict target condition was $15 \mathrm{~ms} /$ item and was not significantly different from zero (Pearson's $r=.3, p=.11$ ). The slope of the conflict distractors condition was $82 \mathrm{~ms} /$ item and was significantly different from zero $(r=.46, p=.01)$. Thus, when searching 


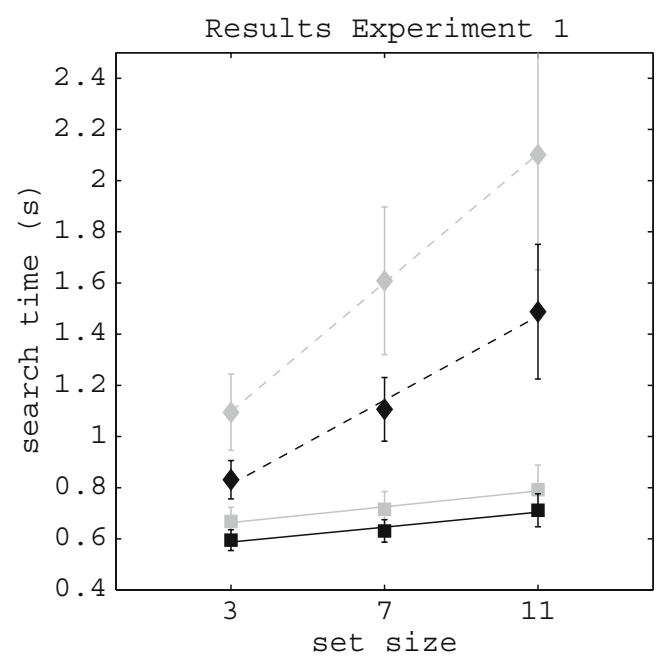

Fig. 2 Results of Experiment 1: Mean search times (y-axis) for 10 observers as a function of set size ( $x$-axis). Target-present data are presented in black, target-absent data in gray. Squares represent search times for the conflict target conditions; solid lines are linear fits for the search times in these conditions. Diamonds represent search times for the conflict distractors conditions; dashed lines are linear fits for the search times in these conditions. Error bars represent standard errors of the means. The results show that search for a conflict target among nonconflict distractors is more efficient than search for a nonconflict target among conflict distractors

for a conflict target among nonconflict distractors, search is faster than when searching for a nonconflict target among conflict distractors.

The results show that interocular conflict leads to a search asymmetry: Searching for a conflict target among nonconflict distractors is much more efficient than vice versa. Using Wolfe's qualification of search performance, the conflict target performance would classify just outside the range of "quite efficient" (Wolfe, 1998).

\section{Experiment 2}

In Experiment 1, we showed that search for a conflict target among nonconflict distractors is faster than search in the opposing environment. However, one might argue that the interocular conflict per se is irrelevant to performance on the search task, and that observers instead search within the fused image of the two images. Observers might therefore be searching the fused image for a target containing orthogonal orientations among single-orientation distractors, or for a single-orientation target among orthogonal-orientation distractors.

If search for an orthogonal (double) orientation target among single-orientation distractors is faster than search for a single-orientation target among double-orientation distractors, this could explain the search asymmetry observed in Experiment 1. To address this alternative explanation, in
Experiment 2 observers searched for an orthogonalorientation target among single-orientation distractors, or for a single-orientation target among orthogonal-orientation distractors (Fig. 1b). Such an orthogonal orientation element was computed by adding two orthogonal Gabors (with parameters specified in the General method section) and multiplying the resulting pattern by a factor of .5. As a result, the peak contrast of an orthogonal orientation element was the same as the peak contrast of a single-orientation element. Importantly, the display contained no interocular conflict, since the images presented to the two eyes were exactly the same.

Results

We observed no search asymmetry between searching for double-orientation targets among single-orientation distractors and vice versa (Fig. 3). If the search asymmetry in Experiment 1 were caused by faster search for a doubleorientation target among single-orientation distractors than for a single-orientation target among double-orientation distractors, we should also have observed a search asymmetry in this experiment. The slope for the double-target condition was $3 \mathrm{~ms} /$ item and was not significantly different from zero $(r=.24, p=.39)$, and the slope for the single-target condition was $4 \mathrm{~ms} /$ item and was not significantly different from zero $(r=.25, p=.37)$, either. Obviously, the slopes of the two conditions were not significantly different $[t(8)=0.45, p=.33]$.

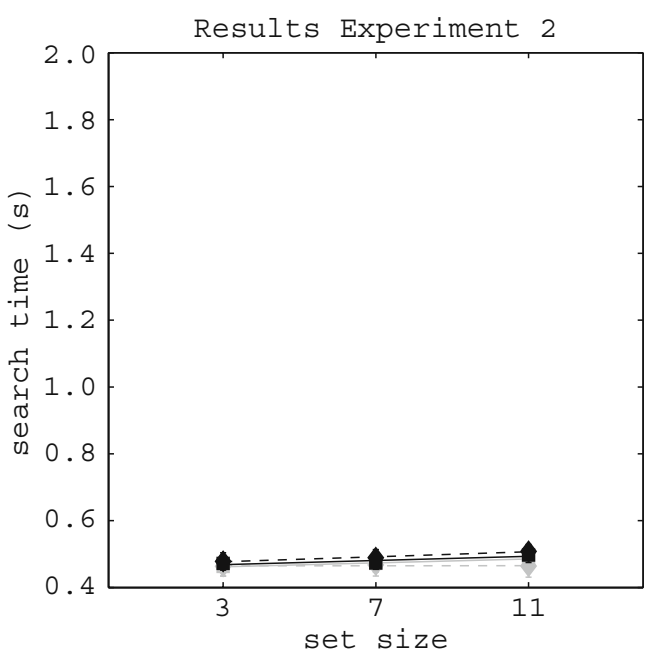

Fig. 3 Results of Experiment 2: Mean search times (y-axis) for 5 observers as a function of set size ( $x$-axis). Target-present data are presented in black, target-absent data in gray. Squares represent search times for the double-target conditions; solid lines are linear fits for the search times in these conditions. Diamonds represent search times for the double-distractors conditions; dashed lines are linear fits for the search times in these conditions. Error bars represent standard errors of the means. The results show that search for a double target among single distractors is as efficient as search for a single target among double distractors 


\section{Experiment 3}

The results of Experiment 2 show that the results of Experiment 1 are unlikely to be due to search on fused images of the displays presented dichoptically. This suggests that interocular conflict is the crucial feature leading to the search asymmetry in Experiment 1 . However, it could still be argued that the target and distractors in Experiment 1 were dissociated by having one or two orientations. In the following experiment, we provide a strong test for the proposed special role of interocular conflict in the observed search asymmetry. Targets and distractors were now dissociated solely by the presence or absence of interocular conflict: Observers searched for an interocular conflict target among nonconflict (double) distractors or for a noninterocular conflict (double) target among interocular conflict distractors (Fig. 1c). If interocular conflict is crucial for the search results of Experiment 1, search should be comparable to the conflict target condition of Experiment 1 . If the conflict is irrelevant, and the images simply fuse, search should become very inefficient (or even impossible), since fusion of the images would lead to the displays consisting solely of double-orientation elements (i.e., the conflict elements would fuse to double-orientation elements).

\section{Results}

The results of Experiment 3 are displayed in Fig. 4. The slope for the conflict target condition was $4 \mathrm{~ms} / \mathrm{item}$ and

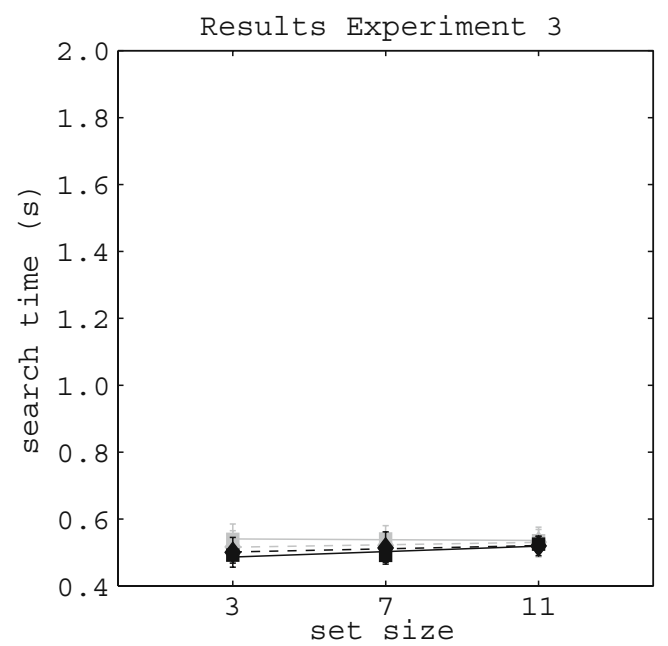

Fig. 4 Results of Experiment 3: Mean search times ( $y$-axis) for 5 observers as a function of set size ( $x$-axis). Target-present data are presented in black, target-absent data in gray. Squares represent search times for the conflict target conditions; solid lines are linear fits for the search times in these conditions. Diamonds represent search times for the conflict distractors conditions; dashed lines are linear fits for the search times in these conditions. Error bars represent standard errors of the means. The results show that search for a conflict target among double distractors is as efficient as search for a double target among conflict distractors was not significantly different from zero $(r=.28, p=.32)$. The slope of the conflict distractors condition was also $2 \mathrm{~ms} /$ item and was also not significantly different from zero $(r=.09, p=.74)$. These results show that search is highly efficient when the presence of interocular conflict is the sole difference between target and distractors. Interestingly, search for a nonconflict target among conflict distractors is also fast and efficient, in contrast to the results of Experiment 1. We return to this issue in the General Discussion.

\section{Experiment 4}

Several aspects of interocular conflict might have caused the search asymmetry observed in Experiment 1. One option is that the conflicting information is somehow detected at an early processing stage, leading to search that approaches efficient search (we comment on this in the General discussion). Another option is that observers use perceptual alternations as a cue to detect a target. As mentioned in the introduction, when each of the two eyes is presented with a different image, the percept alternates between the two images. In the present study, there is conflict at one location in the conflict target condition and at multiple locations in the conflict distractors condition. In the conflict target condition, then, the detection of a single perceptual alternation is sufficient to decide whether a target is present. In the conflict distractors condition, on the other hand, only the absence of a perceptual alternation at a single position is sufficient to decide whether a target is present or not. In the latter case, an observer will therefore need to detect alternations at all positions except one to decide whether a target is present. Clearly, such a strategy would lead to faster search for the conflict target condition than for the conflict distractors condition.

To test whether observers might be using the occurrence of perceptual alternations as a cue to decide whether a search display contains a target, we investigated the time course of perceptual alternation for each observer. Importantly, if observers use perceptual alternations as a cue to decide whether or not a target is present, search times would have to be longer than the time it takes for a perceptual alternation to occur.

In this experiment, a single element was presented below fixation (at the same distance as the elements in the search displays). The parameters of the element were the same as in Experiment 1 (contrast, spatial frequency, etc.). The task of the observer was to continually indicate whether the horizontal or vertical orientation was dominant in perception. Orientation per eye (horizontal or vertical) was counterbalanced. Each observer performed four trials of $60 \mathrm{~s}$. 

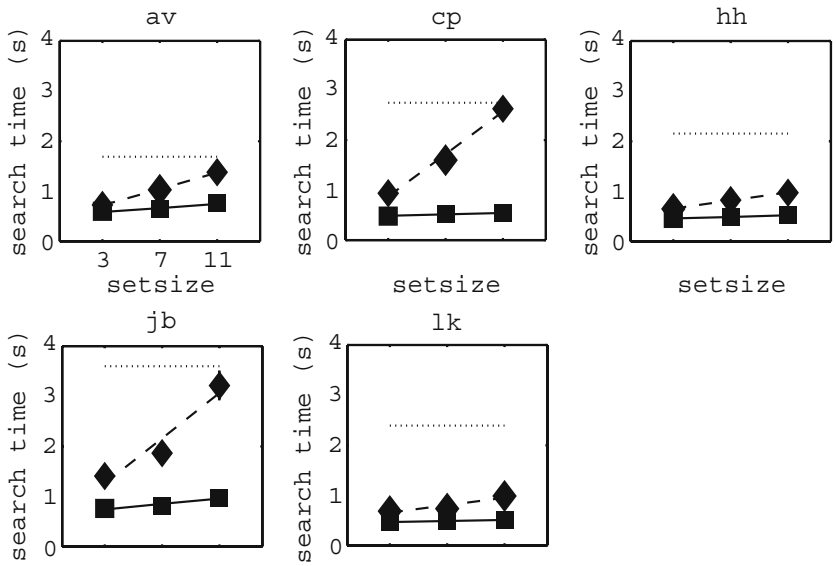

$1 \mathrm{k}$

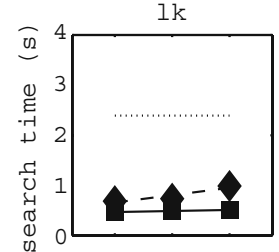

setsize

Fig. 5 Results of Experiments 1 and 4. The dashed and solid lines and the squares and diamonds represent mean reaction times as in Fig. 2, but now for 5 individual observers. The dotted line represents the mean dominance duration for that observer. The results show that mean dominance duration were generally larger than the largest reaction times of Experiment 1

\section{Results}

Figure 5 shows the mean dominance durations (the dotted lines) for 5 observers, with their search results from Experiment 1. The mean dominance durations and standard errors of the means for each observer were $1.7 \mathrm{~s}(0.08)$, $2.7 \mathrm{~s}(0.13), 2.2 \mathrm{~s}(0.12), 3.6 \mathrm{~s}(0.23)$, and $2.4 \mathrm{~s}(0.10)$, respectively. As can be seen in Fig. 5, the search times for individual observers are mostly shorter than their mean dominance durations. Also worthwhile to note is that the mean duration of the first dominance phase also mainly exceeds the search times for the observers. Based on these results, it is therefore highly unlikely that the occurrence of perceptual alternations could have served as a cue to find a target in Experiment 1.

\section{Interim discussion 1}

In a set of four experiments, we have demonstrated so far that search for a target containing interocular conflict is, using
Wolfe's (1998) classification, near "quite efficient" (15 ms/ item), and that search for a conflict target among nonconflict distractors is faster than search for a nonconflict target among conflict distractors. Furthermore, Experiments 2, 3, and 4 eliminated possible alternative explanations that observers might have searched for the fused image or used perceptual alternations as a cue to detect conflict targets. However, the main finding is at odds with the results of Wolfe and Franzel (1988), who performed a similar experiment, but did not find a search asymmetry. In the following three experiments, we investigated the origin of this discrepancy.

Wolfe and Franzel (1988) performed a similar set of experiments in which observers also searched for a target containing interocular conflict among nonconflict distractors, and vice versa. The results of their study are, however, quite different from the ones reported here. First, search for an interocular conflict target was slower (26 $\mathrm{ms} /$ item) than reported here. In addition, the authors did not find a search asymmetry for interocular conflict: Search for a conflict target among nonconflict distractors was not faster than for a nonconflict target among conflict distractors (26 vs. $32 \mathrm{~ms} / \mathrm{item}$, respectively). What could be the reason that the results of Wolfe and Franzel differ from the ones reported here? As can be observed from Table 1, there are quite a few differences between setup, stimuli, and design used in our study and that of Wolfe and Franzel. In the next three experiments, we seek to find the factor that dissociates the two sets of findings.

\section{Experiment 5}

The goal of Experiment 5 was to replicate the results of Wolfe and Franzel (1988). We therefore matched the stimulus and procedure of their study as closely as possible. Wolfe and Franzel presented images dichoptically using a rotating shutter that produced $30-\mathrm{Hz}$ flicker. To simulate this, stimuli were switched on and off at a rate of $30 \mathrm{~Hz}$ (a display was alternately presented to the left and the right eye). The elements consisted of maximum-contrast square-
Table 1 Differences between Wolfe and Franzel (1988) and the present study

\begin{tabular}{lll}
\hline & Experiments 1 and 3 & Wolfe and Franzel \\
\hline Setup & Mirror stereoscope & Rotating shutter \\
Refresh rate & $85 \mathrm{~Hz}$ & $30 \mathrm{~Hz}$ \\
Background & Gray & Black \\
Search elements & Gabors $(4 \mathrm{cpd})$ & Square wave gratings $(3.1 \mathrm{cpd})$ \\
Element eccentricity & $3 \mathrm{deg}$ & 4 deg \\
Target-present level & $50 \%$ & $75 \%$ \\
Set size & 3,7, and 11 & 2,4 , and 8 \\
\hline
\end{tabular}


Fig. 6 The stimuli used in Experiments 5 and 6 (a) and Experiment 7 (b). In the conflict target condition (left panels), one of the elements contained line orientations that were orthogonal for the two eyes. In the conflict distractors conditions (right panels), all of the elements except one had orthogonal orientations for the two eyes

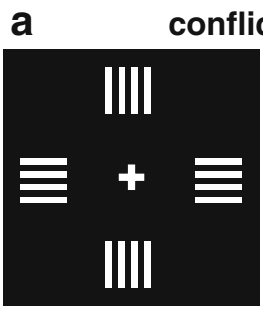

left eye's image

b

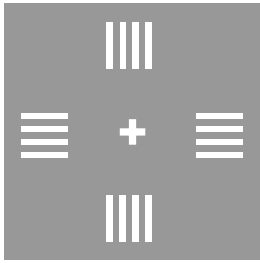

left eye's image

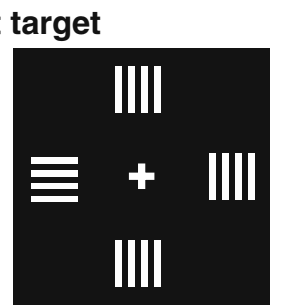

right eye's image

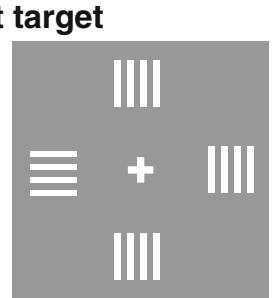

right eye's image

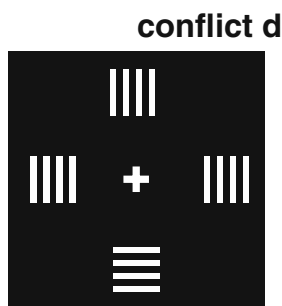

left eye's image

conflict distractors

left eye's image

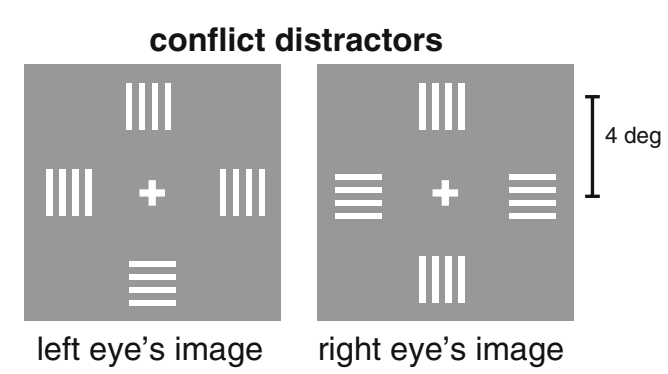

right eye's image

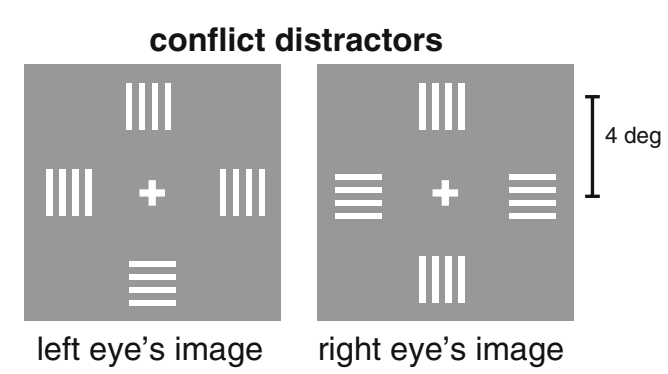

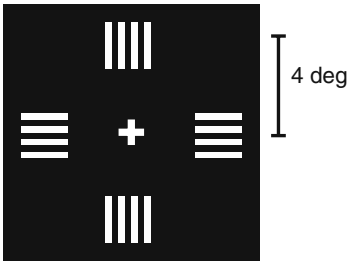

right eye's image wave gratings with a spatial frequency of $3.1 \mathrm{cpd}$ presented on a black background. Elements were placed $4^{\circ}$ from the fixation cross and had a size of $1.6^{\circ}$.

In parallel with Experiment 1, search displays again consisted of two basic configurations (see Fig. 6). In the first configuration, the orientation of the target for the left eye was orthogonal to that for the right eye, and the orientations for the distractors were the same for each eye (conflict target condition). In the second configuration, the orientation for the target was the same for the left and right eyes, and the orientations of the distractors for the left eye were orthogonal to the orientations for the right eye (conflict distractors condition). The set sizes used for this experiment were 2,4 , and 8 . A total of $50 \%$ of the trials contained a target.

\section{Results}

The results of Experiment 5 replicate the findings of Wolfe and Franzel (1988): We observed no search asymmetry (Fig. 7), nor did we observe search as fast as in our Experiment 1 for a conflict target. Search for the conflict target condition led to a slope of $40 \mathrm{~ms} /$ item. The slope for this condition was significantly different from zero $(r=.51$, $p=.004)$. Search for the conflict distractors led to a slope of $43 \mathrm{~ms} /$ item. This slope was also significantly different from zero $(r=.48, p=.007)$. The slope of the conflict distractors condition was not higher than that of the conflict target condition $[t(18)=0.26, p=.4]$.

What aspect of the search display caused the discrepancy between the results of Wolfe and Franzel (1988) and Experiment 4, on the one hand, and those of Experiment 1, on the other?

\section{Experiment 6}

A possible candidate to explain the discrepant results of Experiments 1 and 5 is the $30-\mathrm{Hz}$ flicker in the stimuli of Wolfe and Franzel (1988) and Experiment 5. A 30-Hz flicker is-relative to the $85 \mathrm{~Hz}$ in Experiments 1, 2 and 3 -quite intrusive. It is possible that the $30-\mathrm{Hz}$ flicker

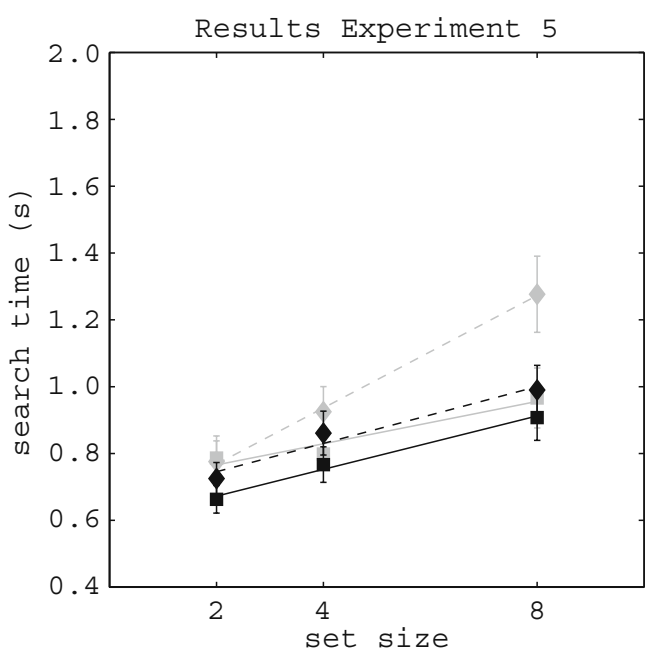

Fig. 7 Results of Experiment 5: Mean search times (y-axis) for 10 observers as a function of set size ( $x$-axis). The target-present data are presented in black, the target-absent data in gray. Squares represent the search times for the conflict target conditions; the solid lines are linear fits for the search times in these conditions. Diamonds represent the search times for the conflict distractors conditions; the dashed lines are linear fits for the search times in these conditions. Error bars represent standard errors of the means. The results show that search for a conflict target among nonconflict distractors is more efficient than search for a nonconflict target among conflict distractors. However, search for a conflict target is not efficient, nor is there a significant search asymmetry 
masks the degree to which the conflict target stands out from the nonconflict distractors, leading to steeper search slopes. In Experiment 6, we tested this prediction. The stimuli and procedure were identical to those of Experiment 5, except that the stimuli were presented at the standard monitor refresh rate of $85 \mathrm{~Hz}$ instead of $30 \mathrm{~Hz}$.

\section{Results}

The results of Experiment 6 were highly similar to those of Experiment 5, ruling out flicker rate as a possible explanation for the observed search differences in Experiment 1 (Fig. 8). Search in the conflict target condition led to a slope of $31 \mathrm{~ms} /$ item. The slope for this condition was not significantly different from zero $(r=.32, p=.24)$. Search in the conflict distractors condition led to a slope of $48 \mathrm{~ms} / \mathrm{item}$. This slope was significantly different from zero $(r=.63, p=$ $.01)$. The slope of the conflict distractors condition was not significantly higher than that of the conflict target condition $[t(8)=1.26, p=.12]$.

\section{Experiment 7}

The results of Experiment 6 show that $30-\mathrm{Hz}$ flicker is not responsible for the absence of a search asymmetry for interocular conflict. Another difference between the search display

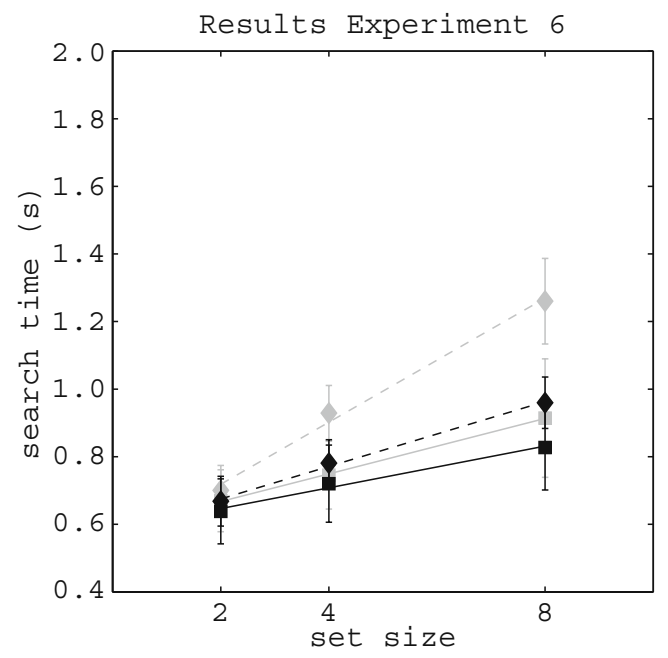

Fig. 8 Results of Experiment 6: Mean search times (y-axis) for 5 observers as a function of set size ( $x$-axis). The target-present data are presented in black, the target-absent data in gray. Squares represent search times for the conflict target conditions; solid lines are linear fits for the search times in these conditions. Diamonds represent search times for the conflict distractors conditions; dashed lines are linear fits for the search times in these conditions. Error bars represent standard errors of the means. The results are qualitatively similar to those of Experiment 5. Thus, removing the flicker from the stimulus did not lead to efficient search in the conflict target condition, nor did it lead to a search asymmetry used by Wolfe and Franzel (1988) and the display in Experiment 1 is the difference in contrast: In their experiments, the elements were white stripes on a black background, whereas in Experiment 1, we presented Gabors on a gray background. In Experiment 7, we address whether search for interocular conflict could be contingent on stimulus-background contrast. The stimuli and procedure were identical to those in Experiment 6, with the exception that background luminance was raised from black to gray $\left(20.4 \mathrm{~cd} / \mathrm{m}^{2}\right)$.

\section{Results}

The results are displayed in Fig. 9. Search for the conflict target led to a slope of $13 \mathrm{~ms} /$ item. This slope was not significantly different from zero $(r=.27, p=.15)$. Search for the nonconflict target led to a slope of $58 \mathrm{~ms} /$ item. This slope was also not significantly different from zero $(r=.26, p=.16)$. It might appear odd that this large slope was not significantly different from zero, but this was due to the large variability in slopes between observers. Importantly, though, the slope of the conflict distractors condition was significantly larger than that of the conflict target condition [t(18) $=1.87, p=.04]$.

\section{Interim discussion 2}

Our Experiment 7 revealed that the critical parameter in search for interocular conflict is luminance contrast. In

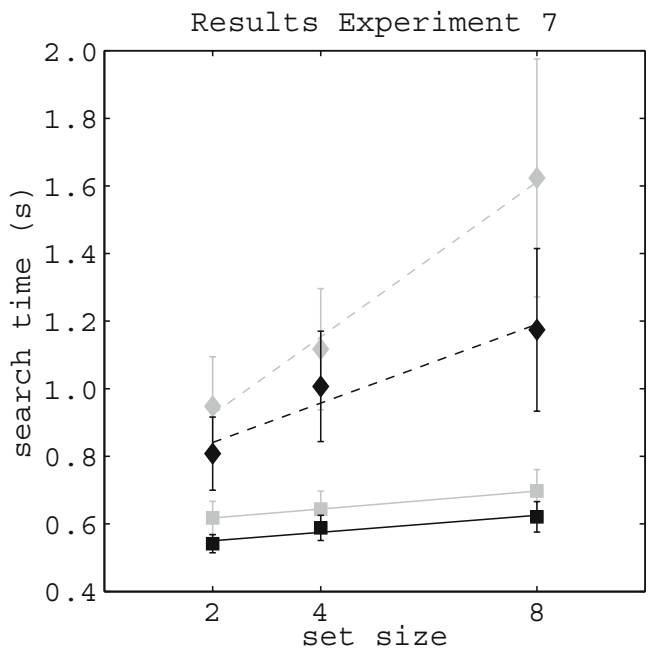

Fig. 9 Results of Experiment 7: Mean search times (y-axis) for 10 observers as a function of set size ( $x$-axis). Target-present data are presented in black, target-absent data in gray. Squares represent search times for the conflict target conditions; solid lines are linear fits for the search times in these conditions. Diamonds represent search times for the conflict distractors conditions; dashed lines are linear fits for the search times in these conditions. Error bars represent standard errors of the means. The results show that efficient search occurs for the conflict target condition when the contrast is lowered. In addition, lowering the contrast leads to a search asymmetry 
Experiments 5 and 6, the contrast of the search elements with the background was high, leading to inefficient search for interocular conflict as well as the absence of a search asymmetry. In Experiments 1 and 7, the contrast of the elements with the background was much lower. As a result, search for interocular conflict became more efficient and a search asymmetry appeared. Paradoxically then, lowering the contrast of a stimulus containing interocular conflict increases the conspicuity of the conflict. What can be the cause of this surprising result? In other words, since lowering the contrast of elements usually lowers their conspicuity, why is this relation reversed for interocular conflict?

Before we propose an answer to this question, we return to the search based on fused elements, which was tested in Experiment 2. Search for orthogonal targets among singleorientation distractors (mimicking search based on fused elements) was not faster than search for single-orientation targets among orthogonal distractors. This result does not exclude, however, the possibility that fusion could have played a role in the efficient search for interocular conflict. As Wolfe (1983) has shown, briefly presented dichoptic stimuli are fused when the presentation time does not exceed $150 \mathrm{~ms}$. After this limit, the dichoptic stimuli will start to engage in binocular rivalry (Wolfe, 1983). As can be seen from our results, the search times for all experiments fall in the range that would cause binocular rivalry to dominate over fusion. Still, it might be possible that the elements appeared to be fused at the onset of a trial, and that observers could base their search on the fused image. From this, we hypothesize that search was based on abnormal fusion of conflicting images. The fusion was abnormal, because "normal" fusion would have led to the situation tested in Experiment 2: search for a doubleorientation among single-orientation distractors. As the results of Experiment 2 show, properly fused images do not lead to the search asymmetry observed in Experiment 1 .

Crucially, the hypothesis that search was based on abnormally fused elements can also explain why a search asymmetry was observed with a gray background (our Exps. 1 and 7), but not when using a dark background (our Exps. 5 and 6; Wolfe \& Franzel, 1988). In studies on binocular rivalry, it is known that the contrast of the images strongly influences the dynamics: When the contrast of both images is lowered, the rate of perceptual alternations will decrease (e.g., Brascamp, van Ee, Noest, Jacobs, \& van den Berg, 2006; Levelt, 1968). In addition, when the contrast of two orthogonal gratings is sufficiently lowered, they will appear to be fused (Liu, Tyler, \& Schor, 1992). In the latter study, observers indicated when orthogonal gratings "disintegrated" from appearing to be fused to appearing to be engaged in rivalry. The time it took for images to start rivaling depended on the contrast: At high contrast, disintegration started at about $200 \mathrm{~ms}$. With decreasing contrast, this time increased gradually up to about $15 \%$ contrast, at which point the duration of the orthogonal percept increased by about a factor of 2 . This finding could explain why search performance in our experiments was dependent on contrast: When contrast is high (Exps. 5 and 6; Wolfe \& Franzel, 1988), abnormal fusion will be weak, and as a result, targets and distractors will look alike, leading to the absence of a search asymmetry. When the contrast of the elements with the background is lower (Exps. 1 and 7), however, abnormal fusion will be stronger, and targets and distractors will be easier to discriminate. As a result, a search asymmetry can be observed.

\section{Experiment 8}

In this final experiment, we explicitly tested the "abnormal fusion" account put forward above. That is, we hypothesized that the conspicuity of interocular conflict is increased when abnormal fusion of incompatible images is optimal. We therefore used the stimuli of Experiment 1, but presented them for just $150 \mathrm{~ms}$. According to Wolfe (1983), abnormal fusion occurs during this first period of interocular conflict. If abnormal fusion is accountable for the high conspicuity of interocular conflict, a target containing the conflict should be easily found, even with this short presentation time.

The stimuli in this experiment were identical to those of Experiment 1, except that the displays were erased from the screen after $150 \mathrm{~ms}$. Set sizes and the numbers of targetpresent and -absent trials were also identical, as well as the blocked presentation of conflict target and nonconflict target trials. The task for the observers was similar: Indicate whether or not the display contained a target. Since the stimuli were erased after $150 \mathrm{~ms}$ and lots of incorrect responses were to be expected, the task was not speeded.

\section{Results}

We calculated $d^{\prime}$ (sensitivity) for detecting the target at each set size for the conflict target and nonconflict target conditions (Fig. 10). Next, we performed a repeated measures ANOVA, with nature of the target and distractors (two levels) and set size (three levels) as factors. The analysis showed main effects of nature of the target $[F(1,4)=$ $25.0, p=.008]$ and set size $[F(2,3)=15.0, p=.03]$, but no significant interaction. This shows that sensitivity for detecting the target was much higher in the conflict target conditions than in the nonconflict target condition. In addition, it shows that sensitivity decreased with increasing set size. Next, we analyzed the degree to which sensitivity for detecting a target differed from maximum sensitivitythat is, when sensitivity was optimal (all hits and no false alarms). Since optimal sensitivity leads to a sensitivity that is 


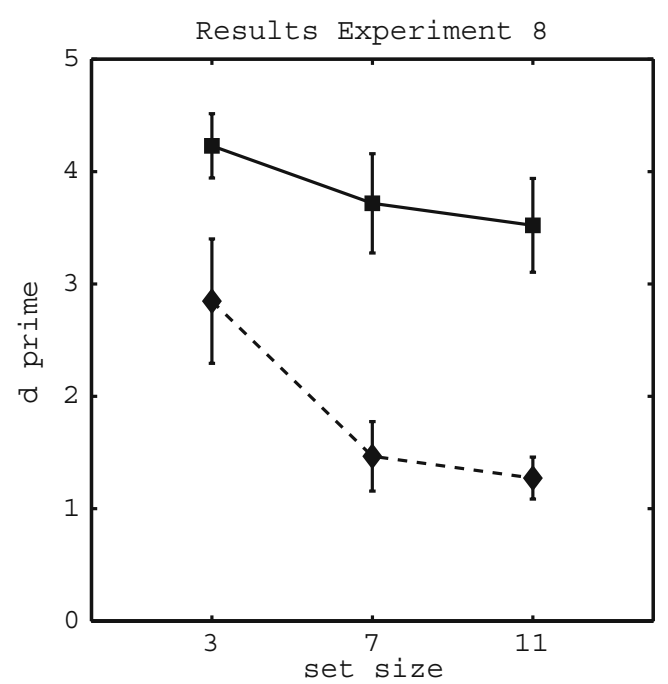

Fig. 10 Results of Experiment 8: Sensitivity $\left(d^{\prime}\right)$ for detecting a conflict target among nonconflict distractors (black squares, solid lines) and for detecting a nonconflict target among conflict distractors (black diamonds, dashed lines). Sensitivity for detecting the conflict target was much higher than for detecting a nonconflict target. In addition, sensitivity for detecting either target decreased with increasing set size

infinite, we truncated fraction hits and false alarms at .99 , leading to a maximum $d^{\prime}$ of 4.7. One-sample $t$ tests showed that sensitivity was not lower than optimal for the lowest two set sizes in the conflict target condition $[t(4)<2.1, p>.05]$. For all of the other conditions, sensitivity was below optimal [conflict target, set size 11: $t(4)=2.7, p=.03$; nonconflict target, set sizes 5, 7, and 11: $t \mathrm{~s}(4)>3.3, p<.05]$. Even though sensitivity for detecting the conflict target was below optimal for set size 11 (sensitivity dropped to a still considerable value of 3.5), these results show that observers are very good at detecting a conflict target when it is present for just $150 \mathrm{~ms}$.

\section{General discussion}

We investigated the conspicuity of interocular conflict in a set of eight experiments and demonstrated that interocular conflict can approach efficient search behavior and can lead to a search asymmetry. According to the qualification of search behavior by Wolfe (1998), search in Experiments 1 and 7 would just fall outside the range of "quite efficient." Furthermore, we ruled out two alternative explanations and reconciled our findings with previous work by Wolfe and Franzel (1988), identifying an important role for stimulus contrast in the effect of interocular conflict on visual search behavior. Finally, in Experiment 8 we showed that search for interocular conflict is about optimal when the display is present for just $150 \mathrm{~ms}$, supporting our hypothesis that abnormal fusion is the driving force behind the modulation of conspicuity of interocular conflict.

In trying to find the cause of the results described above, we considered several candidate explanations. A first candidate, perceptual alternations, was ruled out in Experiment 4: The mean percept duration (perceptual dominance) for the elements we used exceeded the search times for all observers. Thus, observers could not use perceptual alternations as a cue to detect a target.

A second candidate, which we did not consider in our experiments, was binocular luster. Although Wolfe and Franzel (1988) did not find evidence for efficient search for binocular rivalry, they did report efficient search for binocular luster (slope was $4.9 \mathrm{~ms} /$ item). Binocular luster occurs when a dark field is presented to one eye and a light field to the other. When confronted with such a stimulus, the observer typically reports perceiving a metallic appearance (Dove, 1851; von Helmholtz, 1924). Were the relatively fast searches in Experiments 1, 7, and 8 also caused by binocular luster? Although we did not ask the observers to describe the sensation of the elements in the search display, there are several reasons indicating that luster did not play a role in our experiments. First, luster has been described as occurring only for stimuli exceeding $1^{\circ}$ (Howard \& Rogers, 2002; Pieper \& Ludwig, 2001). In our Experiment 1, the size of the Gaussian envelope of the Gabor elements was $0.3^{\circ}$. There was thus no reason for us to assume that binocular luster occurred in that experiment. Second, binocular luster is strongest with large luminance differences between two patches presented dichoptically (e.g., a bright white disk to one eye and a dim black disk to the other; Levelt, 1968). In our Experiment 1, the average luminance of both (interocular) Gabor elements was identical. Thus, binocular luster is not a good candidate to describe our results.

Also, we considered the possibility that fusion of the images was driving our search results. In Experiments 2 and 3 , we showed that a fused version of interocular conflict cannot be responsible for our results. However, as argued above, we hypothesized that abnormal fusion, occurring during the first phase of presenting interocular conflict, was responsible. Our Experiment 8 provided evidence that sensitivity for detecting interocular conflict is about optimal when the conditions for abnormal fusion also are. Interestingly, our findings show that the conspicuity of interocular conflict is not determined by "proper" fusion (like binocular fusion of nonrival images), but by abnormal fusion, occurring during the first phase of interocular conflict.

This hypothesis about abnormal fusion is in line with the claim that "the prerivalry experience is not simply binocular fusion" (Solomon, John, \& Morgan, 2006, p. 1488). For example, observers can easily discriminate between fused gratings and those that are optically fused (Georgeson \& 
Meese, 1997). In experiments by Solomon et al., a display with orthogonal gratings was used in which the orientation of left-eye target elements was the same as the orientation of the right-eye distractors. When element density was high, observers could indicate whether the target region was located on the left or the right side within $250 \mathrm{~ms}$. This finding suggests that - as argued here-short presentation of images with interocular conflict leads to abnormal fusion (dubbed "proto-rivalry" by Solomon et al., 2006).

Moreover, although we did not ask our observers about the phenomenal appearance of the displays, inspection of the stimuli used (we invite readers to free-fuse the stimuli in Fig. 1a) reveals that the phenomenological appearance of the conflict target is quite different from that of a properly fused state (Fig. 1b). This corroborates our notion that the elements fuse abnormally and that there is something special about interocular conflict in visual search.

The results of Experiment 3 also support the claim that abnormal fusion (or "proto-rivalry") leads to the search behavior reported here. In this experiment, fusion of the elements would lead to all the elements having a similar appearance, which should have led to inefficient search behavior (i.e., large set size effects). The fact that observers could still find the (interocular conflict) target very rapidly (search was largely independent of set size) strongly supports our claim for a special status of interocular conflict. Note that, at first sight, the results for the conflict distractors condition of Experiment 3 are in conflict with the results for that condition in Experiment 1. Whereas we observed a significant set size effect in Experiment 1 (a slope of $82 \mathrm{~ms} / \mathrm{item}$ ), we did not find this effect in Experiment 3 (slope of $4 \mathrm{~ms} /$ item). However, this difference in search behavior can easily be explained by the nature of the information on which the search could be performed. In Experiment 1, the target and distractors could only be dissociated on a binocular level: Binocular combination of the monocular information would reveal whether there was interocular conflict in the element or not (see Fig. 1a). As a result, target identity was not available on a monocular level. In contrast, target identity was available on a monocular level in Experiment 3 (Fig. 1c). From this observation, the conclusion emerges that when target identity is available on a monocular level, search is fast and efficient (both conditions of Experiment 3). When target identity can only be established on a binocular level, search is (1) fast, approaching efficient search, for interocular conflict among nonconflict distractors, and (2) slower and inefficient for noninterocular conflict among conflict distractors. Note that this reasoning also applies to the results of Experiment 2: Target identity there could be established on a monocular level, resulting in fast and efficient search behavior.

To conclude, then, we have shown that interocular conflict can be conspicuous and leads to a search asymmetry when the conditions for abnormal fusion are optimal. The present study also shows that there is early access to monocular signals, a result that is in line with the "proto-rivalry" observed by Solomon et al. (2006), as well as with the finding that an ocular singleton (a singleton defined by eye of origin) can play a role during visual search (Zhaoping, 2008).

Author Note C.L.E.P. is supported by a VENI grant from the Netherlands Organization for Scientific Research (NWO).

Open Access This article is distributed under the terms of the Creative Commons Attribution Noncommercial License which permits any noncommercial use, distribution, and reproduction in any medium, provided the original author(s) and source are credited.

\section{Appendix}

Table 2 Overview of the observers used in the experiments

\begin{tabular}{lllllllll}
\hline Observer & Exp. & $\begin{array}{l}\text { Exp. } \\
2\end{array}$ & $\begin{array}{l}\text { Exp. } \\
1\end{array}$ & $\begin{array}{l}\text { Exp. } \\
4\end{array}$ & $\begin{array}{l}\text { Exp. } \\
5\end{array}$ & $\begin{array}{l}\text { Exp. } \\
6\end{array}$ & $\begin{array}{l}\text { Exp. } \\
7\end{array}$ & $\begin{array}{l}\text { Exp. } \\
8\end{array}$ \\
\hline A.H. & & & & & & & $\mathrm{x}$ & \\
A.R. & $\mathrm{x}$ & & $\mathrm{x}$ & & $\mathrm{x}$ & & $\mathrm{x}$ & \\
A.V. & $\mathrm{x}$ & $\mathrm{x}$ & & $\mathrm{x}$ & & & & \\
C.L.E.P. & $\mathrm{x}$ & $\mathrm{x}$ & $\mathrm{x}$ & $\mathrm{x}$ & $\mathrm{x}$ & $\mathrm{x}$ & $\mathrm{x}$ & $\mathrm{x}$ \\
H.H. & $\mathrm{x}$ & $\mathrm{x}$ & & $\mathrm{x}$ & $\mathrm{x}$ & $\mathrm{x}$ & & \\
I.B. & $\mathrm{x}$ & & & & $\mathrm{x}$ & & $\mathrm{x}$ & \\
J.S.B. & $\mathrm{x}$ & $\mathrm{x}$ & & $\mathrm{x}$ & & $\mathrm{x}$ & & \\
J.V. & & & & & $\mathrm{x}$ & $\mathrm{x}$ & & \\
L.K. & $\mathrm{x}$ & $\mathrm{x}$ & & $\mathrm{x}$ & $\mathrm{x}$ & $\mathrm{x}$ & & \\
M.B. & $\mathrm{x}$ & & $\mathrm{x}$ & & $\mathrm{x}$ & & $\mathrm{x}$ & \\
M.S. & & & & & & & $\mathrm{x}$ & \\
R.H. & $\mathrm{x}$ & & $\mathrm{x}$ & & $\mathrm{x}$ & & $\mathrm{x}$ & $\mathrm{x}$ \\
R.P. & & & & & & & & $\mathrm{x}$ \\
S.M. & & & & & & & $\mathrm{x}$ & \\
S.P. & & & & & & & $\mathrm{x}$ & \\
S.S. & & & & & & & & $\mathrm{x}$ \\
T.V. & & & & & & & & $\mathrm{x}$ \\
T.W. & $\mathrm{x}$ & & $\mathrm{x}$ & & $\mathrm{x}$ & & $\mathrm{x}$ & \\
\hline & & & & & & & \\
\end{tabular}

\section{References}

Alais, D., \& Blake, R. (2005). Binocular rivalry. Cambridge: MIT Press.

Blake, R., \& Logothetis, N. K. (2002). Visual competition. Nature Reviews. Neuroscience, 3, 13-21. 
Brainard, D. H. (1997). The psychophysics toolbox. Spatial Vision, $10,433-436$

Brascamp, J. W., van Ee, R., Noest, A. J., Jacobs, R. H. A. H., \& van den Berg, A. V. (2006). The time course of binocular rivalry reveals a fundamental role of noise. Journal of Vision, 6, 12441256.

Dove, H. W. (1851). Ueber die Ursachen des Glanzes und der Irradiation, abgeleitet aus chromatischen Versuchen mit dem Stereoskop. Poggendorffs Annalen, 83, 169-183.

Georgeson, M. A., \& Meese, T. S. (1997). Perception of stationary plaids: The role of spatial filters in edge analysis. Vision Research, 37, 3255-3271.

von Helmholtz, H. (1924). Treatise on physiological optics (J. P. C. Southall, Trans.). Rochester: Optical Society of America. Original work published 1909.

Howard, I. P., \& Rogers, B. J. (2002). Tokens for stereopsis. In Seeing in depth: Vol. 2. Depth perception (pp. 101-141). Toronto: Porteous.

Levelt, W. J. (1968). On binocular rivalry. The Hague. The Netherlands: Mouton.

Liu, L., Tyler, C. W., \& Schor, C. M. (1992). Failure of rivalry at low contrast: Evidence of a suprathreshold binocular summation process. Vision Research, 32, 1471-1479.

Neisser, U. (1967). Cognitive psychology. New York: Appleton-CenturyCrofts.

Panum, P. L. (1858). Physiologische Untersuchungen über das Sehen mit zwei Augen. Kiel: Schwerssche Buchhandlung.

Pieper, W., \& Ludwig, I. (2001). Binocular vision: Rivalry, stereoscopic lustre, and sieve effect. Perception, 30(Suppl), 75-76.
Solomon, J. A., John, A., \& Morgan, M. J. (2006). Monocular texture segmentation and proto-rivalry. Vision Research, 46, 1488-1492.

Treisman, A. M., \& Gelade, G. (1980). A feature-integration theory of attention. Cognitive Psychology, 12, 97-136.

Treisman, A., \& Gormican, S. (1988). Feature analysis in early vision: Evidence from search asymmetries. Psychological Review, 95, 1548.

Treisman, A., \& Souther, J. (1985). Search asymmetry: A diagnostic for preattentive processing of separable features. Journal of Experimental Psychology: General, 114, 285-310.

Wolfe, J. M. (1983). Influence of spatial frequency, luminance, and duration on binocular rivalry and abnormal fusion of briefly presented dichoptic stimuli. Perception, 12, 447-456.

Wolfe, J. M. (1994). Guided Search 2.0: A revised model for visual search. Psychonomic Bulletin \& Review, 1, 202-238.

Wolfe, J. M. (1998). Visual search. In H. Pashler (Ed.), Attention (pp. $13-$ 73). Hove: Psychology Press.

Wolfe, J. M., \& Franzel, S. L. (1988). Binocularity and visual search. Perception \& Psychophysics, 44, 81-93.

Wolfe, J. M., \& Horowitz, T. S. (2004). What attributes guide the deployment of visual attention and how do they do it? Nature Reviews. Neuroscience, 5, 495-501.

Wolfe, J. M., Cave, K. R., \& Franzel, S. L. (1989). Guided search: An alternative to the feature integration model for visual search. Journal of Experimental Psychology: Human Perception and Performance, 15, 419-433.

Zhaoping, L. (2008). Attention capture by eye of origin singletons even without awareness - A hallmark of a bottom-up saliency map in the primary visual cortex. Journal of Vision, 8(5), 1:1-18. 